\title{
ACTEMRA $^{\circledR}$ (tocilizumab), solution injectable par voie sous-cutanée
}

Limitation de I'utilisation d'ACTEMRA ${ }^{\circledR}$ (tocilizumab) stylo prérempli aux patients atteints de AJIs/AJIp, âgés de 12 ans et plus, en raison d'un risque éventuel d'injection intramusculaire chez les patients âgés de moins de 12 ans.

En accord avec Swissmedic, Roche Pharma (Suisse) SA souhaite vous informer de nouvelles informations importantes concernant la sécurité d'ACTEMRA ${ }^{\circledR}$ (tocilizumab):

Un article de synthèse de la littérature spécialisée a décelé des indices indirects d'un risque éventuel d'injection intramusculaire chez des patients âgés de moins de 12 ans atteints d'une arthrite juvénile idiopathique systémique (AJls) ou d'une arthrite juvénile idiopathique polyarticulaire (AJIp). Aucun cas n'a cependant été rapporté dans l'ensemble de la base de données de sécurité de Roche.

\section{Mesures}

Par mesure de sécurité, I'utilisation d'ACTEMRA ${ }^{\circledR}$ stylo prérempli a désormais été limitée aux patients âgés de 12 ans et plus. Les patients atteints d'AJIs/AJlp, âgés de moins de 12 ans qui sont traités actuellement par ACTEMRA ${ }^{\circledR}$ en stylo prérempli doivent maintenant utiliser la seringue préremplie (SPR) ou la forme injectable par voie intraveineuse (i.v.), ces deux formes étant appropriées pour les enfants âgés de moins de 12 ans.

\section{Informations contextuelles}

La forme injectable par voie s.c. de tocilizumab est disponible en seringue préremplie (SPR) avec un dispositif de sécurité de l'aiguille ou en stylo prérempli. Une recherche réalisée par Roche dans la littérature spécialisée a montré des indices indirects d'un risque potentiel d'injection intramusculaire chez les patients atteints d'AJIp ou d'AJIs âgés de moins de 12 ans, d'une part, car la couche de tissu souscutané peut être plus mince chez ceux-ci et d'autre part, en raison de la longueur de l'aiguille exposée et de la nécessité d'un angle de 90 degrés lors de l'injection avec ACTEMRA ${ }^{\circledR}$ stylo prérempli.

Ce risque potentiel d'injection intramusculaire lors de l'utilisation du stylo prérempli chez les patients atteints d'AJIp/AJIs, âgés de moins de 12 ans, signifie donc que cette option n'est pas appropriée pour l'administration d'ACTEMRA ${ }^{\circledR}$ dans ce groupe d'âge.

En accord avec Swissmedic, Roche va actualiser I'information professionnelle d'ACTEMRA ${ }^{\circledR}$. Vous trouverez le texte complet de la circulaire de Roche Pharma (Suisse) SA directement sur la page d'accueil du site de Swissmedic dans les communications relatives à la sécurité des médicaments.

\section{Annonce d'effets indésirables}

Pour le signalement de tout effet indésirable (EI), Swissmedic recommande d'utiliser le portail d'annonce développé à cet effet. L'utilisation de l'Electronic Vigilance System (EIViS) permet de faire la déclaration des El par saisie directe. Toutes les informations nécessaires à ce sujet sont disponibles sur www.swissmedic.ch 